\title{
Unimanual, Bimanual and Bilateral Weight Perception of Virtual Objects in the Master Finger 2 Environment
}

\author{
Christos D. Giachritsis, Pablo Garcia-Robledo, Jr., Jorge Barrio, Alan M. Wing and Manuel Ferre, \\ Member, IEEE
}

\begin{abstract}
Human users can obtain information about the physical properties of an object through direct manipulation with one or two hands. Object manipulation of virtual objects using force feedback haptic interfaces is very challenging due to current technological constrains that often affect the information obtained by the user. Here, we describe the Master Finger 2 (MF2), a force feedback device which allows manipulation of one or more objects with one or two hands. We use experimental data to evaluate the performance of MF2 based on its capability to simulate effectively the weight of virtual objects. The results and implications for system design are discussed.
\end{abstract}

\section{INTRODUCTION}

$\mathrm{W}$ HEN we explore an object with our touch we use cutaneous and proprioceptive feedback to obtain information about its physical properties including size, shape, material, surface texture, temperature and weight. Some of these properties such as size, shape and material could be directly obtained through vision while properties such as temperature and weight require the sense of touch to be perceived accurately [1].

Haptic interfaces, which are based on a robot-arm design, use force feedback to simulate size, shape and weight. The most common of these devices have one-contact-point, therefore, inherently allowing object exploration only through sequential touching. In addition, object manipulation is restricted in lifting and displacing the object in an unrealistic way since the simulated object has to 'stick' on the finger that lifts, thus, effectively ignoring the displacement of centre of mass. A solution to this problem is to combine more than one-contact-point devices to allow for more naturalistic object manipulation including grasping and rotation [2]. One of the

Manuscript received 1 April, 2010. (Write the date on which you submitted your paper for review.) This work was supported by the European Commission under the IMMERSENCE Integrated Project of the Sixth Frame Program (IST-4-027141-IP).

C. D. Giachritsis* and A. M. Wing are with the University of Birmingham, Edgbaston, Birmingham, B15 2TT (corresponding author*, phone: 0044-121-414-7260; fax: 0044-121-414-xxxx; e-mail: c.giachritsis@bham.ac.uk).

P. Garcia-Robledo, J. Barrio and M. Ferre are with the Universidad Politecnica de Madrid, Centro de Automática y Robótica (UPM-CSIC), C\ Jose Gutierrez Abascal, 2. 28006. Madrid. most common problems with the multi-one-contact-designs is that the workspace inevitably reduces dramatically since more than one devices are involved [3].

Here, we describe a new multifinger haptic interface which allows grasping and manipulation of objects with one and two hands: the Master Finger 2 (MF2). The MF2 also includes a graphic interface which shows the position of object and hands during manipulation. In addition, we report experiments which were designed to evaluate the effectiveness of MF2 in simulating object weight during unimanual, bimanual, and bilateral object manipulation. In section 2, the design of MF2 is explained; in section 3, two experiments that were conducted to evaluate the MF2 are described; in section 4 , the results are reported and in section 5 , we discuss the findings and the implications for system design.

\section{MASTER FINGER 2}

\section{A. Mechanical Design}

The haptic device used is called MasterFinger-2 [4]. The mechanical structure of the MasterFinger-2 haptic interface was designed to ease object manipulation within a virtual environment. It comes with a modular design of two fingers with six Degrees of Freedom (DoF) per finger: three DoF for movement of the hand and three for finger rotation. Every

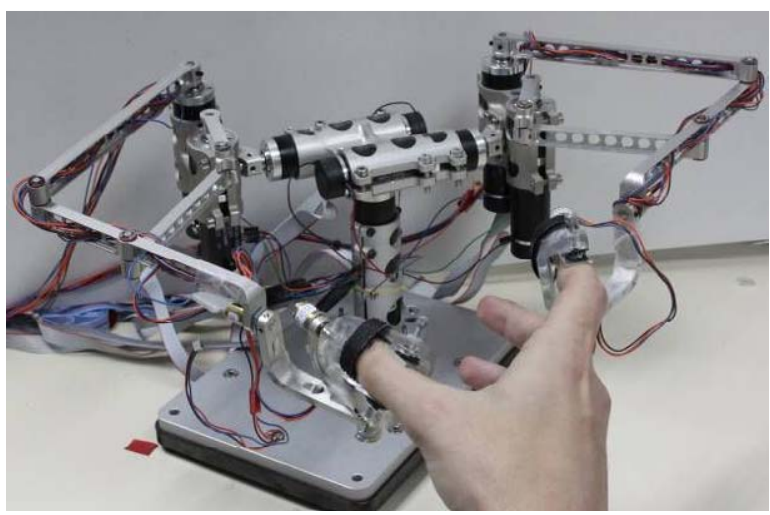

Fig. 1. MasterFinger-2 Haptic Device placed over a table. User can insert his or her thumb and index fingers into the thimbles in order to manipulate and feel virtual environments. 


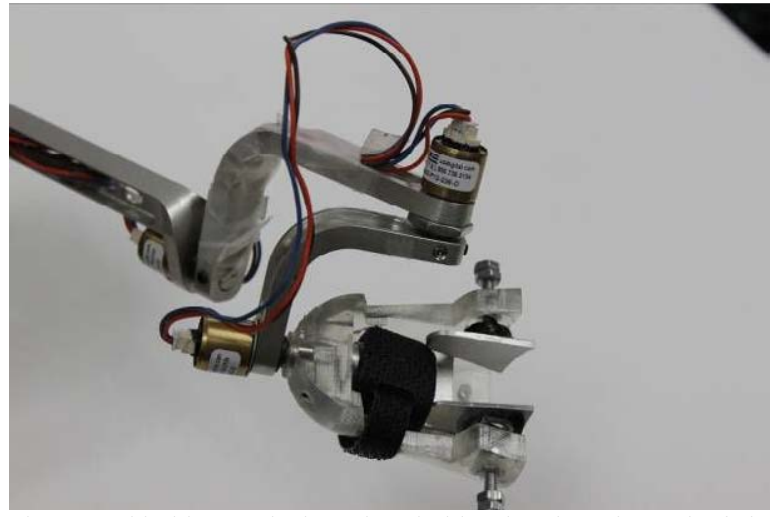

Fig. 2. Thimble attached to the gimble placed at the end of the parallel-series structure. The thimble is attached to the finger by using the screws to press the metal sheets.

finger has its own mechanical structure and electronic controller. The haptic device consists of a three DoF parallelseries structure per finger and it is actuated by three electric motors. The parallel series allows reflecting forces in all directions, but not torques. Fig. 1 provides a general view of this two-finger haptic device.

In order to determine if the haptic interface works properly, it is important to focus on the capability of the device to move freely within the workspace. This is a mandatory issue to be regarded since it defines how the operator can interact with the virtual environment. Depending on the requirements for application, some characteristics of the haptic device, such as transmission of motion and force exertion at the end of the kinematics chain will have an effect on the performance of the device. It will ease or stop driving the motion.

The ensemble of the two finger's mechanical structures is connected to the haptic device base using an additional joint. This additional joint significantly increases the MF-2 workspace in comparison to a two fingered interface made up of two haptic devices. Thanks to this additional joint the mechanical structure can turn avoiding collisions between the links. As a result of this additional freedom of movement, very realistic haptic interactions have been achieved. The operator is able to grasp and move different objects in the virtual environment [5].

A gimble is placed at the end of the parallel-series structure. It is made up of two links and three rotational axes. It allows the thimble to achieve any orientation without constraints on the finger movement. The three rotational axes intersect at the finger tip, which are also aligned with the last link of the haptic interface. This geometrical configuration ensures that the haptic interface reflects forces at the finger tip at any direction without torque components. These orientation angles are obtained by encoders located at the gimble's rotational axes. MA3 encoders from USDigital were used for measuring the positions of the axes. The MA3 is a miniature rotary absolute magnetic shaft encoder that reports the shaft position over $360^{\circ}$ with no stops or gaps.

The apparent weight of the designed mechanical system is $79.62 \mathrm{~g}$ per finger at a distance of $30 \mathrm{~cm}$ from the base, while the apparent weight of the Phantom Premium 1.0 is $75 \mathrm{~g}$.

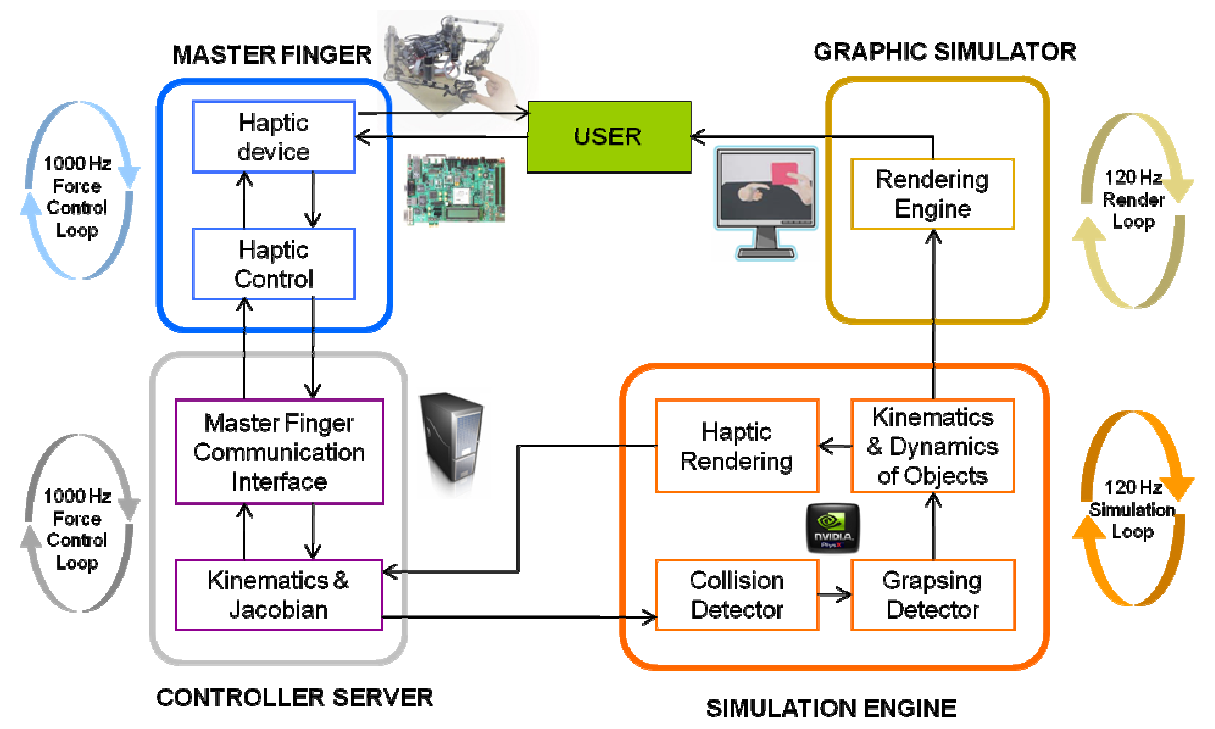

Fig. 3. Distributed Control Architecture. On top left the MasterFinger Haptic Device includes controller to render forces. The devices (as many as needed) are attached to a controller server that calculates Kinematics \& Jacobian on a Linux OS. This server sends position and orientation of all fingers to the Simulation Engine to obtain the forces that must be exerted over the fingers. Finally, the virtual environment is presentd in aa many OpenGL graphic simulators as needed. 


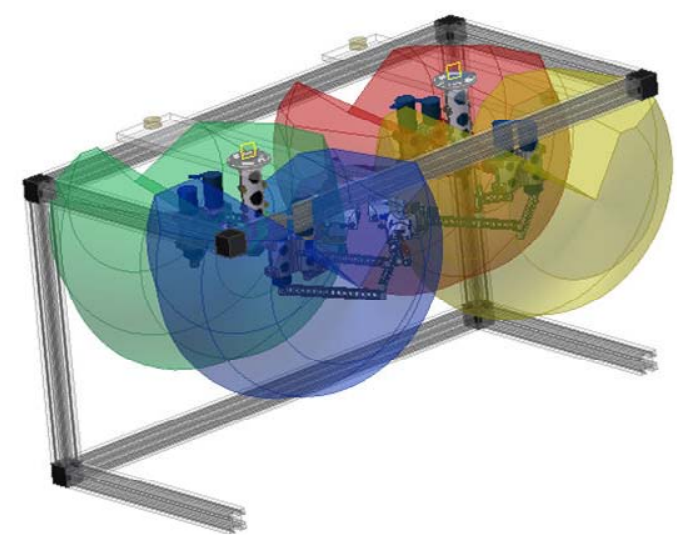

Fig. 4. Set up for a bimanual task and workspace of each finger of each MF-2 regarding 6-DoFs. The distance between both MF-2 can be set as needed depending on the application.

\section{B. Thimbles}

The thimbles are attached to the gimble so as to allow the user to insert his or her thumb and index finger (Fig. 2). The role of the thimbles is twofold. First, the thimble ensures that the operator's finger will not hurt or release when a force is applied. Second, the contact sensors are placed on the thimble so as to measure the force exerted by the operator. The finger rests on aluminum plates which enclosed the FlexiForce sensors. The sensors were placed between two plates in order to ensure that all forces applied are evenly distributed in all the sensor area.

The thimble was designed to insert the largest finger possible into it. Then the thimble is adjusted to the user's finger size by using two side screws that are gently fixed to both sides of the finger.

\section{Control Architecture}

MasterFinger-2 has a modular and scalable design (Fig. 3). The system can be used with as many haptic interfaces and screens as needed, depending on the application. Each MasterFinger haptic device has a Virtex-5 FPGA (ML-505 board) per finger. This board has the low level control of the system programmed on the PowerPC. The PowerPC runs the low level controller under a VxWorks real time operating system to assure a constant rate. With this system, the achievable bandwidth when reflecting forces is $150 \mathrm{~Hz}$.

All MasterFingers must be connected via Ethernet to a Linux OS server which is in charge of the kinematics and jacobian calculus that cannot be processed on the FPGA due to high computational cost. This server provides information of position and orientation of the final end effectors, as well as the filtered data measured by the contact sensors. This controller also computes the gravity precompensation. It also

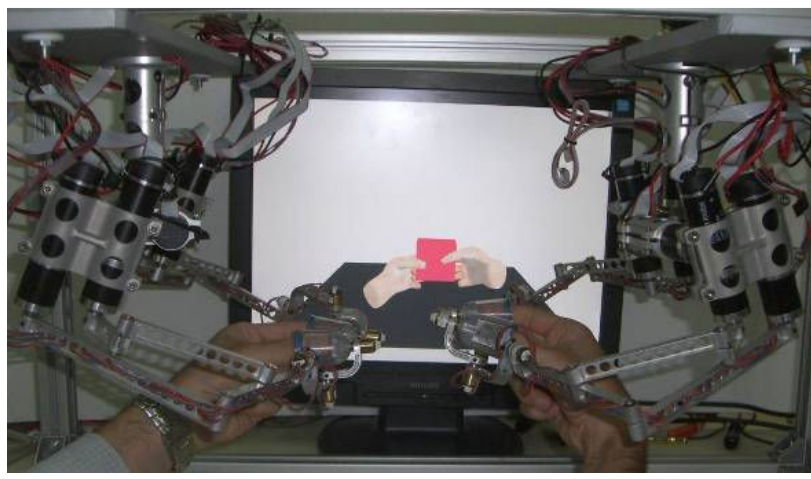

Fig. 5. MF 2 setup for bimanual and bilateral manipulation. Operator uses his or her thumb and index fingers to carry out the manipulation of a block.

includes an antiwindup subsystem to guarantee safety measures. The control is based on an impedance control. The controller server is connected to the simulation engine via Ethernet. The simulation engine is based on nVidia's PhysX engine. First of all, the engine evaluates when a collision between the fingers and the object occurs based on PhysX calculation. Second, a grasping detector detects when a grasping condition takes place and, consequently, the movement of the fingers actuates in a different manner over the objects.

Finally, an OpenGL simulation engine sends the positions and orientations of all fingers to the Graphic Simulator in order to display the virtual environment.

\section{EXPERIMENTAL SETUP}

\section{A. Master Finger 2: Two-hand manipulation}

The operator commonly has to use both hands in order to carry out complex tasks. A setup was designed with two MF-2 haptic interfaces that are placed in an inverted fashion such that the user is provided with considerable workspace for carrying out the manipulation.

Both MF-2 are attached to a metallic structure. The dimension of the structure sets the limits of distance between the two MF-2 bases. When an object is manipulated with four fingers the fingers become constrained due to the geometry and size of the manipulated object. Consequently, the distance between bases is adjusted beforehand in accordance with the size of the objects to be manipulated. Fig. 4 shows the workspace of each finger when the two MF-2 are placed in the structure. When an object is manipulated with both hands only the center of the structure is used, but when each hand is handling a different object the hand is free to move around the joint of the base.

The progress of the virtual grasping task is shown to the 
TABLE I

STIMULUS PRESENTATION AND RESPONSE IN A SingLE TRIAL

\begin{tabular}{lll}
\hline \hline & Condition 1: RH-RH & Condition 2: RH-BH \\
\hline Phase 1 & Lift test/std with RH & Lift test/std with RH/BH \\
Phase 2 & Lift std/test with RH & Lift std/test with BH/RH \\
Phase 3 & Which weight felt heavier? (first or second?) \\
\hline
\end{tabular}

user on a computer display. The right and left hands as well as the object manipulated are presented to the user. Hand postures are controlled by the movement of haptic devices. The posture of each virtual hand is calculated according to the position and orientation of the index and thumb thimbles. Therefore a perfect correlation between the user movements and the graphical simulation of the task is achieved.

The force exerted to the operator is based on Hooke's law, so it is proportional to the finger-object penetration when a collision is detected. The user also feels an additional vertical force that corresponds to the load of the virtual object. Whenever an object is lifted by only one hand the load is shared by the thumb and the index finger $(50 \%$ of load per finger). However, when grasping is done by both hands, the load is then distributed accordingly, which means $25 \%$ of block load per finger.

\section{B. Experiment 1: Unimanual vs Bimanual Weight Perception}

The aim of this experiment was to evaluate how users perceive the weight of virtual objects during unimanual and bimanual manipulation in the MF2 environment. A twointerval-forced-choice (2IFC) constant stimuli procedure was used with seven weights ranging from $75 \mathrm{~g}$ to $525 \mathrm{~g}$ and step size of $75 \mathrm{~g}$ resulting in a standard weight of $300 \mathrm{~g}$. Two conditions were used: in the first condition, users judged heaviness with the right hand $(\mathrm{RH})$ only and in the second condition, they judged heaviness between $\mathrm{RH}$ and both hands

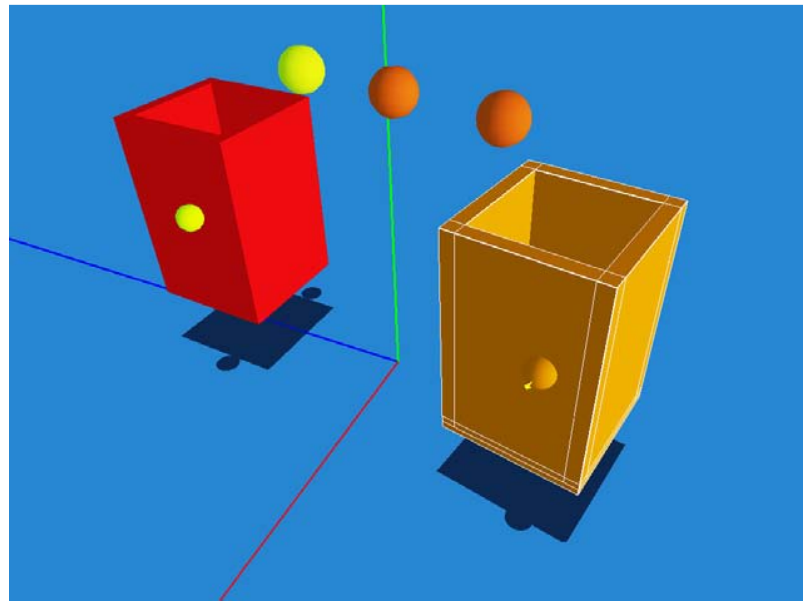

Fig. 6. Graphical representation of the transfer of material from one container to the other, in Experiment 2. During the transfer, the ball changes colour.
(BH). Table 1 shows how stimuli were presented in each trial. The orders of stimulus presentations and manipulation modes were balanced and trials were randomized. Twelve responses per weight were collected.

\section{Experiment 2: Bilateral Sensitivity to Opposite Weight Changes}

The aim of this experiment was to investigate first, how users perceive opposite changes in virtual weight between two objects held in the left hand ( $\mathrm{LH}$ ) and $\mathrm{RH}$, and second, how grip forces between $\mathrm{LH}$ and $\mathrm{RH}$ are adjusted to these changes.

Two virtual non-transparent and equally heavy cubical containers contain a material that initially (i.e., at the beginning of each trial) has the same mass in both containers. Thus, the total weight of both containers and material is exactly the same. When the trial begun, the participants lifted the two containers off the table and held them at about $100 \mathrm{~mm}$ height from the table. After $1.5 \mathrm{~s}$ one of the containers was flashing to signal that part of its content (represented by a green ball) would be transferred to the other container. In mid-air, the ball representing the transferred content changed colour to red in order to facilitate user's response in the third stage (Fig. 6). In the third stage, the participant had to indicate which weight change (e.g., ball), not container, was heavier.

The initial weight of the two containers was $300 \mathrm{~g}$ and three transferred weights were used: 75,150 and $225 \mathrm{~g}$. Based on these weights two combination were used: 75-150 g and 75-225 g. The presentation of weights was balanced. Participants completed 12 trials per combination.

\section{RESULTS}

In Experiment 1, individual and overall psychometric functions were obtained by fitting the logistic rule,

$$
F(x ; \alpha, \beta)=\frac{1}{1+e^{-(x-\alpha) / \beta}},
$$

where $\alpha$ is the location of the function on the $\mathrm{x}$-axis and $\beta$ is its slope. A change in parameter $\alpha$ between unimanual and bimanual lifting would indicate a change in the subjective perception of heaviness while a change in the parameter $\beta$ would indicate a change in sensitivity to weight changes when lifted with one and two hands.

Results showed that virtual weights lifted with the both hands felt lighter than virtual weights lifted with the right hand: $\alpha$ of the RH-BH function shifted (Fig. 7). However, this effect was statistical significant only when the right hand lifted the standard weight in the RH-BH condition $\left(\mathrm{T}_{(4)}=\right.$ 
$3.148 ; \mathrm{p}=0.035)$. In addition, users' sensitivity to changes of virtual weights reduced in the RH-BH condition: $\beta$ of the $\mathrm{RH}-$ $\mathrm{BH}$ function reduced. This deterioration of sensitivity was statistically significant when both hands were used to lift the standard $\left(\mathrm{T}_{(4)}=5.218 ; \mathrm{p}=0.006\right)$ and test $\left(\mathrm{T}_{(4)}=-3.442 ; \mathrm{p}=\right.$ $0.026)$ weight. These results were very similar with ones obtained using real weights [1].

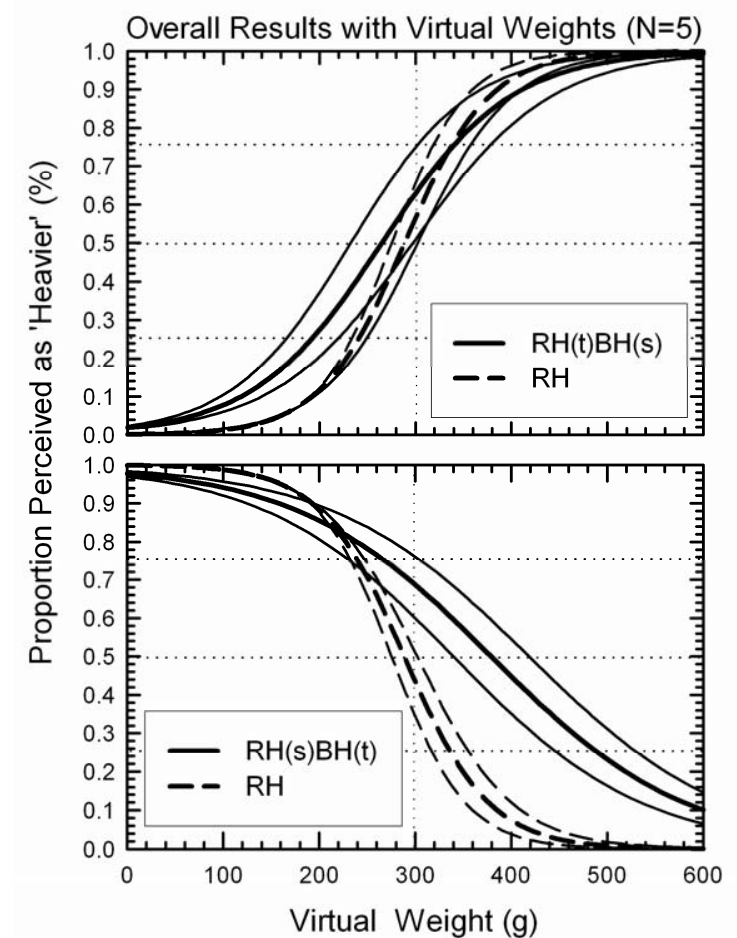

Fig. 7. Overall psychometric function obtained for the experimental (continuous lines) and control (dashed lines) conditions under both presentation orders of the virtual weights. The bold lines represent the average functions and thin lines represent $95 \%$ confidence limits of the two functions (adapted from [6]).

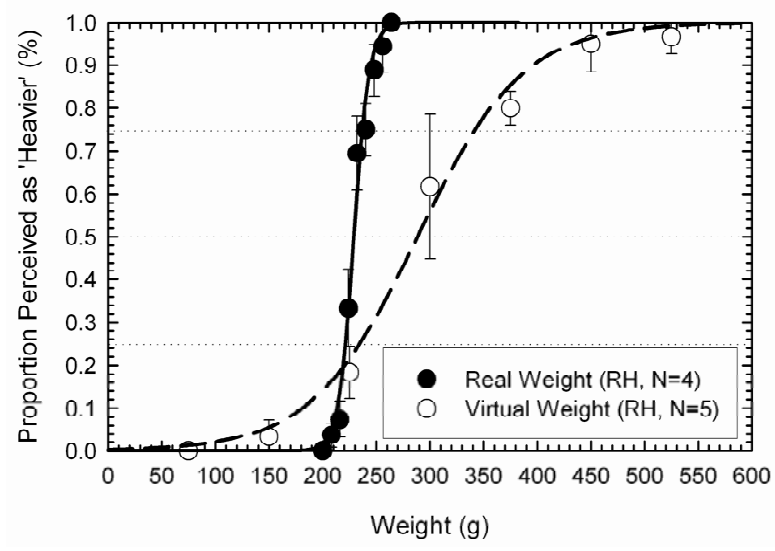

Fig. 8. Comparison of weight discrimination with the right hand $(\mathrm{RH})$ ahen lifting real (black disks) and virtual (white disks) weights. Continuous and dashed curves represent the pshychometric functions for the real and virtual data, respectively.
Further analysis showed that weight discrimination with one hand is better with real than virtual weights (Fig 8; adapted from [6]). In a study which compared human sensitivity to real and virtual weight changes [6], it was found that with real weight users need only $8 \mathrm{~g}$ change to detect a difference while they need a $48 \mathrm{~g}$ change to detect the difference between two virtual weights. Weber's Fraction indicated that weight sensitivity with real weights $(\mathrm{WF}=0.033)$ was nearly five times better than with virtual weights $(\mathrm{WF}=0.161)$.

In Experiment 2, results were very inconsistent. Most of the participants seemed to have found the task of judging the magnitude of the 'gained' and 'lost' weights between the two containers very difficult. For most participants, performance slightly improved when they judged the change from 75-225 g compared to 75-150 g. However, even with double the difference the judgments were nearly at chance level (Fig.9).

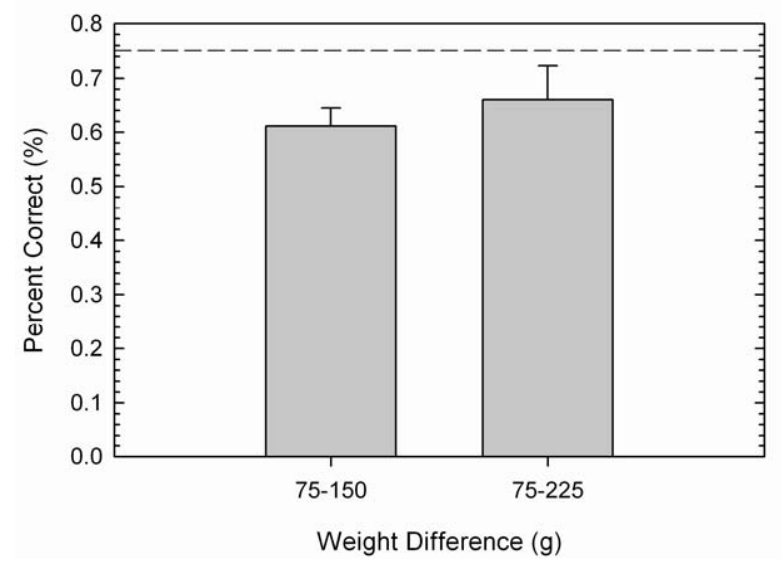

Fig.9. Overall $(\mathrm{N}=6)$ performance in judging the relative heaviness of the lost and gained mass between the two hands in Experiment 2.

Nonetheless, even though it seemed very difficult to judge correctly the magnitude of weight change in the two containers, the grip force seems to respond to changes in virtual weight: that is, users' increase of the grip force appears to follow the sudden increase of donward forces simulating virtual weight (Fig. 10). 

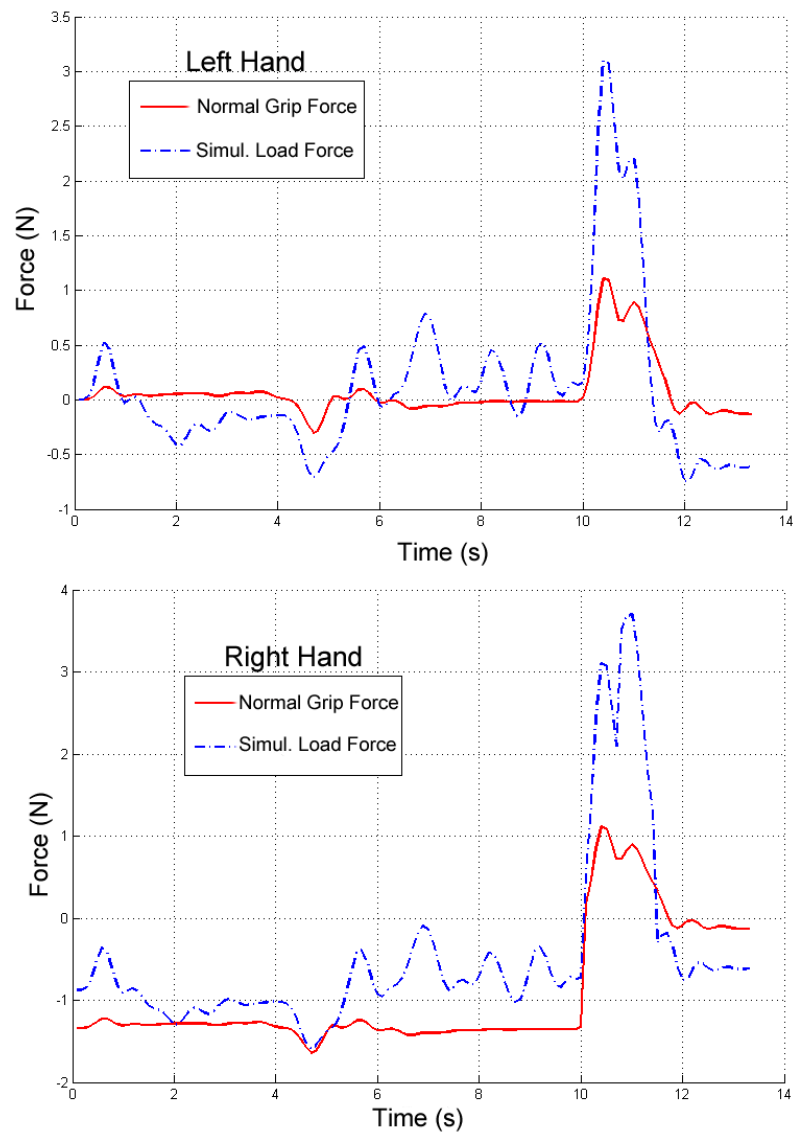

Fig.10. Example of Left Hand (loss) and Right Hand (gain) normal forces (red line) applied by a user during a single trial. It seems that bilateral grip forces respond to vertical forces applied by the MF2

\section{DISCUSSION}

\section{A. Simulation of Bimanual Weight Perception}

In principle, the experiment on discrimination of virtual weights lifted with one and two hands showed that users exhibit the same bias with real weights: unimanually lifted virtual objects feel heavier than bimanually lifted virtual objects with the same simulated weight. This demonstrates that the MF2 haptic interface, through effective delivery of downward forces to all four digits, has managed to generate the presence a bimanually lifted object successfully.

\section{B. Users' Sensitivity to Virtual Weights}

The experiment on weight discrimination with one hand has shown that sensitivity to simulated weight chances of virtual objects is worse than with real weights: users are five times more accurate when discriminating real than virtual weights. This result could be due to perception of virtual weights is based, primarily, on proprioceptive feedback while cutaneous feedback is largely absent. A study with real weights has shown that both proprioceptive and cutaneous feedback are equally important in weight perception [7]. Moreover, the small correction of horizontal forces simulating object size could result in horizontal proprioceptive noise, which may interfere with the vertical proprioceptive feedback signaling the virtual weight.

\section{Bilateral Grip Forces and Perception of Opposite Weight Changes}

The last experiment has shown that users found it very difficult to discriminate the magnitudes of virtual weight that changed during transfer from one had to the other. However, their bilateral grip force seems to respond to these changes indicating that users do sense this change even though they cannot perceive its magnitude accurately. Moreover, users seem to adjust their grip when the visual and haptic input signals a change in weight. Given that the model of weight simulation in MF2 (and other haptic feedback devices with thimbles) is based on delivering forces that push the grip downwards, the users would equally be able to lift and hold the virtual objects just by applying an adequate constant force which would require attaching their grip to the virtual object. Nonetheless, they appear to behave as the weight changes in were real demonstrating that MF2 environment generates a presence of virtual mass.

\section{CONCLUSION}

The MF2 multipoint contact haptic interface has demonstrated an effective simulation of bimanual virtual weight perception and the ability to elicit changes in grip forces due to changes in virtual weights. This behaviour is observed with real weights [8] particular important since the design of MF2 and the However, further work is needed to improve the simulated weight resolution and stability of the grip during grasping and lifting so that horizontal propriocetive engagement (signaling object size) is negligible and vertical proprioceptive engagement (signaling weight) is optimal. Addressing these problems would result in a versatile haptic interface that could be used in precision unimanual and bimanual tasks requiring accurate weight perception.

\section{ACKNOWLEDGMENT}

This work is supported by the European Commission under the IMMERSENCE Integrated Project of the Sixth Frame Program (IST-4-027141-IP). 


\section{REFERENCES}

[1] G. Robles-de-la-Torre, "The Importance of the Sense of Touch in Virtual and Real Environments," IEEE Multimedia, vol. 13, no. 3, August 2006.

[2] A. Peer, M. Buss, "A New Admittance-Type Haptic Interface for Bimanual Manipulations" IEEE/ASME Trans. Mechatron., vol. 13, no. 4, pp. 416-428, 2008.

[3] S A Wall, W S Harwin, "Design of a Multiple Contact Point Haptic Interface", in Proc. Eurohaptics Conf., Birmingham, 2001, pp 146148.

[4] M. Monroy, P. García-Robledo, M. Ferre, J. Barrio, J. Ortego, "Advanced Virtual Manipulation based on Modular Haptic Devices," in Int. Symp. Robot Control (SYROCO'09), 2009, pp 111-116.

[5] P. Garcia-Robledo, J. Ortego, J. Barrio, I. Galiana, M. Ferre, R. Aracil, "Multifinger Haptic Interface for Bimanual Manipulation of Virtual Objects," in IEEE Int. Workshop on Haptic Audio Visual Enviroments and Games, 2009, pp 30-35.

[6] C.D. Giachritsis and A.M. Wing. "Unimanual and bimanual weight discrimination in a desktop setup." in Proc. Eurohaptics, Madrid, 2008, M.Ferre, Ed. LNCS series, Berlin Heidelberg, Springer-Verlag, pp. 378-382.

[7] C. Giachritsis, R. Wright, and A. Wing, "The contribution of proprioceptive and cutaneous cues in weight perception: Early evidence for maximum-likelihood integration". in Proc. Eurohaptics, Amsterdam, 2010, A.M.L. Kapers et al., Eds. LNCS series, Berlin Heidelberg, Springer-Verlag, pp. 11-16.

[8] J.R.Flanagan and A.M.Wing, "The Role of Internal Models in Motion Planning and Control: Evidence from Grip Force Adjustments during Movements of Hand-Held Loads", J. Neurosci., vol. 17, no.4, pp.15191528 . 